\title{
A Three-State Markov-Modulated Switching Model for Exchange Rates
}

\author{
Idowu Oluwasayo Ayodeji \\ Department of Mathematics, Obafemi Awolowo University, Ile-Ife 220005, Nigeria \\ Correspondence should be addressed to Idowu Oluwasayo Ayodeji; idowu.sayo@yahoo.com
}

Received 30 July 2016; Revised 26 September 2016; Accepted 5 October 2016

Academic Editor: Wei-Chiang Hong

Copyright ( 2016 Idowu Oluwasayo Ayodeji. This is an open access article distributed under the Creative Commons Attribution License, which permits unrestricted use, distribution, and reproduction in any medium, provided the original work is properly cited.

\begin{abstract}
Several authors have examined the long swings hypothesis in exchange rates using a two-state Markov switching model. This study developed a model to investigate long swings hypothesis in currencies which may exhibit a $k$-state $(k \geq 2)$ pattern. The proposed model was then applied to euros, British pounds, Japanese yen, and Nigerian naira. Specification measures such as AIC, BIC, and HIC favoured a three-state pattern in Nigerian naira but a two-state one in the other three currencies. For the period January 2004 to May 2016, empirical results suggested the presence of asymmetric swings in naira and yen and long swings in euros and pounds. In addition, taking 0.5 as the benchmark for smoothing probabilities, choice models provided a clear reading of the cycle in a manner that is consistent with the realities of the movements in corresponding exchange rate series.
\end{abstract}

\section{Introduction}

Regime switching models are models which can characterize time series properties in different regimes. Models in which switching among regimes occurs stochastically according to a Markov process are called Markov regime switching models or Markov switching models (MSM) for short. Markov switching model due to Hamilton [1] has been widely applied in economics and finance. Two very popular applications of interest are the studies of long swings in currencies (e.g., Engel and Hamilton [2] and Klaassen [3]) and business cycles (e.g., Garcia and Perron [4]).

First, what are "long swings" and why do they matter in exchange rates? Engel and Hamilton [2] defined "long swings" in dollar as a situation in which "the value of dollar appears to increase in one direction for long periods of time." The study of exchange rate movements has constantly been of interest to econometricians owing to its effects on the nation's growth. Moreover, it is believed that the understanding of exchange rate movements may help avoid potential currency crises. Consequently, lots of efforts have been devoted to developing a theory to describe its process. Since the works of Meese and Rogoff [5], researchers have adopted the random walk hypothesis to explain exchange rate movements until
Engel and Hamilton [2] presented evidence which challenged its continued use. They (Engel and Hamilton) showed that exchange rates switch infrequently between two different states of appreciation and depreciation and consequently could not follow a random walk. Subsequently, a two-state Markov regime switching model was adopted for modeling exchange rates.

Of course, a two-state Markov switching model may provide a satisfactory fit for currencies exposed to a consistent floating exchange rate regime. According to literature, these currencies are expected to appreciate and/or depreciate in response to market forces. See for instance Figure 1 which displayed the exchange rates of US dollars to a unit of euros, British pounds, and Japanese yen. It is easily seen that the three currencies switched between appreciation and depreciation states during the period under review. However, owing to varying monetary policies, currencies which may be allowed to float at some times and pegged at others would definitely exhibit a different pattern. Figure 2 plots the worth of one Nigerian naira (NGN) in US dollars (USD). The plot suggested that naira exhibited some pattern different from other markets examined so far in the literature: in all, three (not two) periods could be identified: depreciation, low appreciation, when 


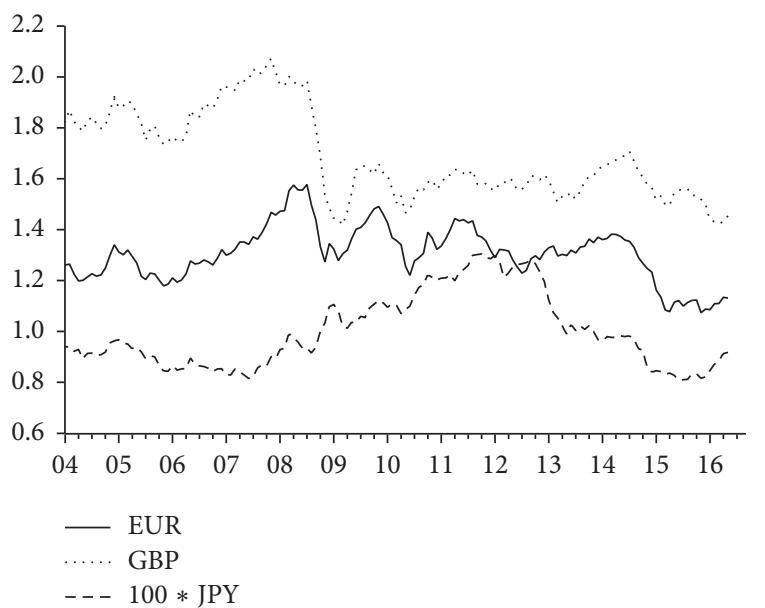

FIGURE 1: Plot of USD/EUR, USD/GBP, and USD/JPY for 2004M012016M05.

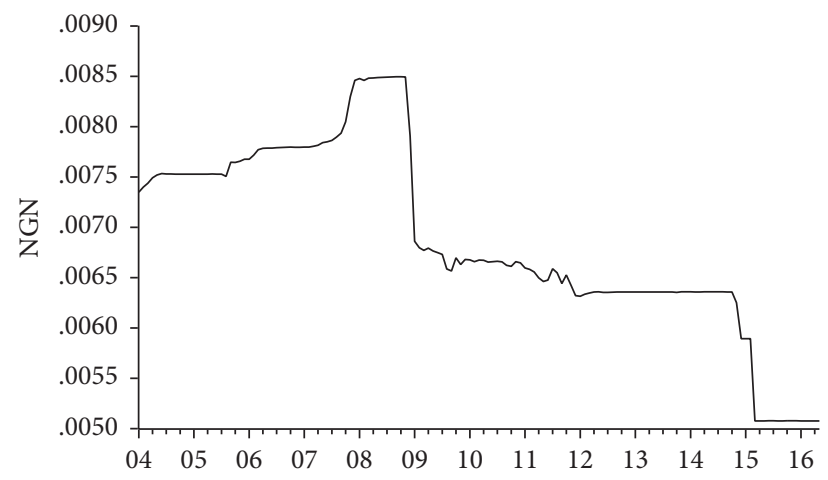

Figure 2: Plot of USD/NGN for 2004M01-2016M05.

there was little or no increase in the number of dollars required to obtain a naira, and high appreciation, when there was substantial increase. Depreciation occurred in the 11th month of 2008 (i.e., 2008M11) to 2009M03 and in 2014M102015M03, while naira gained $0.027 \%$ and $0.068 \%$ against USD between 2005M08-2006M03 and 2007M02-2008M03, respectively. And lastly, the "low appreciation" periods were 2004M06-2005M07, 2006M05-2007M03, 2008M032008M11, 2012M03-2014M10, and 2015M03-2016M05. This peculiar occurrence is easily explained: Nigeria's economy depends heavily on importations which are traded (most often) in US dollars; thus, it is expected that any changes in US currency would bring about reaction ("high appreciation" or "depreciation") in Nigerian naira; and consequently, in such cases, to avoid too much volatilities, the Central Bank of Nigeria (CBN) would usually respond with policies such as pegging naira against dollar, resulting in "low appreciation." A pertinent question to ask is: how does the "long swings" hypothesis apply to a case of more than 2 currency regimes?

The answer to this question is important for several reasons. First, for several other developing and/or importoriented economies which may share this peculiar pattern with Nigeria, it provides a template on the study of long swings hypothesis. Second, it has been shown that government policies affect exchange rate movements. Kaminsky [6], for instance, reported that a change in monetary policy regimes affects the exchange rate depreciation, resulting in long swings. Consequently, information on the existence of long swings is key to exchange rate process determination and policies. And lastly, as is currently been witnessed in Nigeria, for instance, the movements in exchange rate have far-reaching implications for the nation's growth, prices of goods and services, rate of unemployment, purchasing power, balance of payment, import structure, government revenue, economic growth, and other economic factors. Thus, information on long swings may give signals on potential economic recessions and currency crises.

This study will therefore investigate the possibility of the long swings hypothesis in EUR, GBP, NGN, and YEN, all with respect to USD. The $k$-state model to be proposed allows decomposition of exchange rate data into depreciation and appreciation states. These states can be further decomposed into other substates. For instance in the case of naira, in line with Figure 2, the two substates of low appreciation and high appreciation could be created. This is with a view to obtaining regime probabilities which can be used to analyze exchange rates cycle over the selected periods.

The plan of study is as follows: Section 2 discusses the Markov regime switching model. Section 3 presents results on long swings investigation while Section 4 concludes.

\section{Materials and Methods}

Datasets consist of monthly exchange rates for euros, British pounds, Japanese yen, and Nigerian naira over recent period of January 2004-May 2016 (all series are official rates taken from data banks compiled by fxtop.com, which originally collected them from corresponding central banks). Denote $r_{t}=\log \left(e_{t} / e_{t-1}\right)$, the returns on exchange rates $e_{t} \cdot e_{t}$ is the number of dollars per unit of domestic currency.

Consider the following model:

$$
r_{t}=\mu_{S_{t}}+\epsilon_{t}
$$

where

$$
\epsilon_{t} \sim \text { i.i.d. } N\left(0, \sigma_{S_{t}}^{2}\right), \quad S_{t}=1,2, \ldots, k, t=1,2, \ldots, T .
$$

Equation (1) represents the simplest model with switching dynamics. The intercept $\mu$ takes $k$ different values representing the expectations in the $k$ different states, and also the volatilities $\sigma^{2}$ of $\epsilon_{t}$. $S_{t}$ is the unobservable Markov switching variable which evolves according to the following transition probabilities:

$$
P=\left(\begin{array}{cccc}
P_{11} & P_{12} & \cdots & P_{1 k} \\
P_{21} & P_{22} & \cdots & P_{2 k} \\
\vdots & \vdots & \cdots & \vdots \\
P_{k 1} & P_{k 2} & \cdots & P_{k k}
\end{array}\right)
$$


where $P_{i j}=\operatorname{Pr}\left(S_{t}=j \mid S_{t-1}=i\right)$ and

$$
\sum_{j=1}^{k} P_{i j}=1 \quad \forall i
$$
(3) is

The $\log$-likelihood function $(\log L)$ corresponding to (1)-

$$
\log L=\sum_{t=1}^{T} \log f\left(r_{t} \mid S_{t}\right)
$$

where

$$
f\left(r_{t} \mid S_{t}\right)=\frac{1}{\sigma_{S_{t}} \sqrt{2 \pi}} \exp \left\{-\frac{1}{2 \sigma_{S_{t}}^{2}}\left(r_{t}-\mu_{S_{t}}\right)^{2}\right\} .
$$

Since $S_{t}$ are not observable, the estimation process may not be straightforward. Following Hamilton [7], we adopt the following notations:

$$
f\left(r_{t}, S_{t} \mid \Psi_{t-1}\right)=f\left(r_{t} \mid S_{t}, \Psi_{t-1}\right) P\left(S_{\mathrm{t}} \mid \Psi_{t-1}\right),
$$

where $\Psi_{t-1}$ represents the information available up to time $(t-1)$. And

$$
f\left(r_{t} \mid \Psi_{t-1}\right)=\sum_{S_{t}=1}^{k} f\left(r_{t} \mid S_{t}, \Psi_{t-1}\right) P\left(S_{t} \mid \Psi_{t-1}\right)
$$

so that (5) may be updated as

$$
\log L=\sum_{t=1}^{T} \log \sum_{S_{t}=1}^{k} f\left(r_{t} \mid S_{t}, \Psi_{t-1}\right) P\left(S_{t} \mid \Psi_{t-1}\right) .
$$

The estimates of the states' probabilities $P\left(S_{t}=j\right)$ are computed using the following algorithm:

(a) Compute the steady-state probabilities $P\left(S_{1}=1\right.$ । $\left.\Psi_{0}, \ldots, P\left(S_{1}=k \mid \Psi_{0}\right)\right)$.

(b) For $t=2, \ldots, k$,

(i) compute at time $t$ the probability of each state conditional on $\Psi_{t-1}$ as

$$
P\left(S_{t}=j \mid \Psi_{t-1}\right)=\sum_{i=1}^{k} P_{i j} P\left(S_{t}=i \mid \Psi_{t-1}\right) ;
$$

(ii) using the parameters of the models in each state, that is, $\mu_{1}, \mu_{2}, \ldots, \mu_{k}, \sigma_{1}, \sigma_{2}, \ldots, \sigma_{k}$, and the transition probabilities $P_{11}, P_{22}, \ldots, P_{k k}$, compute $\log L$ in each state at time $t$;

(iii) update the probability of each state as

$$
\begin{aligned}
P\left(S_{t}=j \mid \Psi_{t-1}\right) & \\
& =\frac{f\left(r_{t} \mid S_{t}=j, \Psi_{t-1}\right) P\left(S_{t}=j \mid \Psi_{t-1}\right)}{\sum_{j=1}^{k} f\left(r_{t} \mid S_{t}=j, \Psi_{t-1}\right) P\left(S_{t}=j \mid \Psi_{t-1}\right)} .
\end{aligned}
$$

TABLE 1: Comparison of specification measures for selected currencies.

\begin{tabular}{lccccc}
\hline \multirow{2}{*}{ Currency } & $k$ & \multicolumn{4}{c}{ Specification measures } \\
& & $\log L$ & AIC & BIC & HIC \\
\hline \multirow{2}{*}{ EUR } & $2^{*}$ & 353.2494 & -4.6926 & -4.5711 & -4.6432 \\
& 3 & 356.2340 & -4.6518 & -4.4088 & -4.5531 \\
\multirow{2}{*}{ GBP } & $2^{*}$ & 368.2343 & -4.9145 & -4.7724 & -4.8569 \\
& 3 & 365.4255 & -4.7949 & -4.5304 & -4.6875 \\
JPY & $2^{*}$ & 353.4282 & -4.7133 & -4.5709 & -4.6554 \\
& 3 & 350.3974 & -4.5904 & -4.3260 & -4.4830 \\
\multirow{2}{*}{ NGN } & 2 & 652.0555 & -8.7305 & -8.6090 & -8.6811 \\
& $3^{*}$ & 724.0515 & -9.6223 & -9.3793 & -9.5236 \\
\hline
\end{tabular}

${ }^{*}$ Higher log-likelihood and lower criteria values.

Estimation proceeds by finding the set of parameters that maximizes $\log L$ as given in (9).

Sometimes it may be necessary to compute the smoothing probabilities $\left(P\left(S_{t}=j \mid \Psi_{T}\right)\right)$. Kim [8] showed that estimates of the smoothing probabilities may be obtained through a smoothing algorithm, which is a backward iterative process. In addition, expected duration $E[D]$ of a regime is also of utmost importance as it helps to measure the length of stay of the system in a particular state. Denote $D$ the duration of state $j$, and then (Kim and Nelson [9]),

$$
E[D]=\sum_{j=1}^{\infty} j P(D=j) \approx \frac{1}{1-P_{j j}} .
$$

Several statistical packages are available for implementing the switching regression. Examples include Eviews 8.1, R (cran-fMarkovSwitching and MSwM), and MS Regress in Matlab. Though Eviews offers a much simpler interface than $\mathrm{R}$ and Matlab; standard errors of parameter estimates have to be extracted using delta method under Eviews package. This study employed Eviews 8.1.

\section{Results and Discussion}

Table 1 compared the appropriateness of two-state and threestate Markov switching regression for modeling the selected currencies, euro, British pounds, Japanese yen, and Nigerian naira. Specification measures such as the log-likelihood values $(\log L)$, Akaike (AIC), Bayesian (BIC), and HannanQuinn (HIC) information criteria were employed. Recall that

$$
\begin{aligned}
\mathrm{AIC} & =-2 \log L+2 m, \\
\mathrm{BIC} & =-2 \log L+m \log (T), \\
\mathrm{HIC} & =-2 \log L+2 m \log (\log (T)),
\end{aligned}
$$

where $L$ is the likelihood function defined earlier in (9) and $m$ represents the number of parameters included in the corresponding model.

For euros, pounds, and yen, case $k=2$ had the higher log-likelihood values and lower AIC, BIC, and HIC values. Thus, the two states (i.e., $k=2$ ) of appreciation and 
TABLE 2: Maximum likelihood estimates for selected currencies.

\begin{tabular}{|c|c|c|c|c|}
\hline \multirow{2}{*}{ Parameter } & \multicolumn{4}{|c|}{ Currencies } \\
\hline & EUR & GBP & JPY & NGN \\
\hline \multirow{2}{*}{$\mu_{1}$} & -0.026513 & -0.055766 & -0.000156 & -0.086049 \\
\hline & $(0.009956)$ & $(0.022138)$ & $\left(0.002006^{*}\right)$ & $(0.027987)$ \\
\hline \multirow[b]{2}{*}{$\mu_{2}$} & 0.006599 & 0.000226 & 0.001640 & 0.000943 \\
\hline & $(0.002917)$ & $\left(0.001695^{*}\right)$ & $\left(6.21 \times 10^{-5}\right)$ & $\left(0.001060^{*}\right)$ \\
\hline \multirow{2}{*}{$\mu_{3}$} & NA & NA & NA & $4.21 \times 10^{-5}$ \\
\hline & NA & NA & NA & $\left(2.15 \times 10^{-5}\right)$ \\
\hline \multirow{2}{*}{$\sigma_{1}$} & 0.02005381 & 0.01995992 & 0.0232941 & 0.05164954 \\
\hline & $(0.005337342)$ & $(0.01640825)$ & $(0.001391473)$ & $(0.05549624)$ \\
\hline \multirow{2}{*}{$\sigma_{2}$} & 0.01869538 & 0.01871618 & $8.087798 \times 10^{-5}$ & 0.008307131 \\
\hline & $(0.001861891)$ & $(0.001171764)$ & $\left(5.619329 \times 10^{-5}\right)$ & $(0.00054969)$ \\
\hline \multirow{2}{*}{$\sigma_{3}$} & NA & NA & NA & 0.000167884 \\
\hline & $1 \mathrm{~N} A$ & 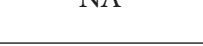 & $1 \mathrm{~N} \Omega$ & $\left(1.29323 \times 10^{-5}\right)$ \\
\hline \multirow{2}{*}{$\beta^{+}$} & \multirow{2}{*}{ NA } & 0.183702 & 0.274173 & \multirow{2}{*}{ NA } \\
\hline & & $(0.073214)$ & $(0.003039)$ & \\
\hline \multirow{2}{*}{$P\left(S_{t}=1 \mid S_{t-1}=1\right)$} & 0.714666 & 0.728827 & 0.963766 & 0.400255 \\
\hline & $(0.03535)$ & $\left(0.47510^{*}\right)$ & (12.9933) & $\left(0.07336^{*}\right)$ \\
\hline \multirow{2}{*}{$P\left(S_{t}=2 \mid S_{t-1}=2\right)$} & 0.919525 & 0.992119 & $6.81 \times 10^{-9}$ & 0.931781 \\
\hline & $\left(0.086041^{*}\right)$ & $(0.007408)$ & $\left(13.48182^{*}\right)$ & $\left(0.12157^{*}\right)$ \\
\hline \multirow{2}{*}{$P\left(S_{t}=3 \mid S_{t-1}=3\right)$} & \multirow[t]{2}{*}{ NA } & \multirow[t]{2}{*}{ NA } & \multirow[t]{2}{*}{ NA } & 0.905627 \\
\hline & & & & $(0.004199)$ \\
\hline
\end{tabular}

${ }^{+} \beta$ is the coefficient of lag 1 (i.e., $r_{t-1}$ ) which was included to correct for autocorrelation in relevant series.

NA means not applicable. Standard errors are in parentheses. ${ }^{*}$ Not significant at $\alpha=0.05$.

depreciation were enough to describe USD/EUR, USD/GBP, and USD/YEN. This, of course, agrees with existing theory that currencies under floating regime arrangement switch infrequently between the states of depreciation and appreciation according to the forces of demand and supply (see also Figure 1). For Nigerian naira, however, it is evident that the three-state model overwhelmingly outperformed the two-state. This is also expected for currency which switches between floating and fixed regimes from time to time according to the monetary policies of its central bank (see also Figure 2).

In what follows, we present empirical estimates for the states favoured by specification measures.

3.1. Investigating the Long Swings Hypothesis. Table 2 displayed maximum likelihood estimates for the four selected currencies.

3.1.1. Case $k=2$. State 1 associated euro with a $2.7 \%$ monthly fall, a $5.6 \%$ fall in British pounds and a $0.016 \%$ fall in Japanese yen. In state 2 , euros experienced greatest rise to the tune of $0.65 \%$ compared to the other two currencies. Except, of course, for yen, state 2 experienced a considerable amount of variability than state 1 . We observed further that the point estimates of $P_{j j}, j=1,2$ were large, ranging from 0.71 to 0.99 , except for Japanese yen which had its $\widehat{P}_{22}=6.81 \times 10^{-3} \%$. These estimated probabilities implied that if the system is in state 1 or 2 , it is likely to remain in that state except for yen whose expected duration in the appreciation state is only 1 month.
Engel and Hamilton [2] posited that exchange rate movements could be characterized by any of following three possibilities: random walk, long swings, or asymmetric swings. If, for Model (1), $\mu_{1}<0$ and $\mu_{2}>0$ and $P_{11}$ and $P_{22}$ are large, then long swings exist. If $\mu_{1}<0,\left|\mu_{1}\right|$ is small and $P_{11}$ is large; in addition, if $\mu_{2}>0$ and large and $P_{22}$ is small, then there is asymmetric persistence in swings. Otherwise, $P_{11}=1-P_{22}$, and exchange rate is said to follow a random walk. Applying this theory to EUR, GBP, and JPY, we observed the following possibilities:

$\widehat{\mu}_{1}=-0.027<0, \widehat{\mu}_{2}=0.0065>0$ and $\widehat{P}_{11}=0.71, \widehat{P}_{22}=$ 0.92 large $\Rightarrow$ long swings for euro.

$\widehat{\mu}_{1}=-0.056<0, \widehat{\mu}_{2}=0.00023>0$ and $\widehat{P}_{11}=0.73$, $\widehat{P}_{22}=0.99$ large $\Rightarrow$ long swings for British pounds.

$\widehat{\mu}_{1}=-0.00016<0, \widehat{\mu}_{2}=0.0016>0$. It is also evident that $\widehat{\mu}_{1}$ is small in absolute compared to $\widehat{\mu}_{2}:(|0.0016|-\mid-$ $0.00016 \mid) \%=0.14 \%$. And lastly, $\widehat{P}_{11}=0.96$ is large while $\widehat{P}_{22}=6.81 \times 10^{-9}$ is very small $\Rightarrow$ asymmetric swings for Japanese yen. Thus, it appears that downward moves for Japanese yen were gradual and drawn out while upward moves were short and sharp.

Finally, Figures 3-5 plotted each domestic currency with its corresponding smoothed probabilities. As is usually the practice, we assumed that there is a switch in regime when the smoothing probability is greater than 0.5 . It is easily seen that the smoothed probability plots presented patterns that match closely with Figure 1 . For instance, pounds fell by $57 \%$ towards the end of 2008. Similarly, the value of euros depreciated from 1.58 to 1.27 between 2008M07 and 2008M11. Notice a similar 

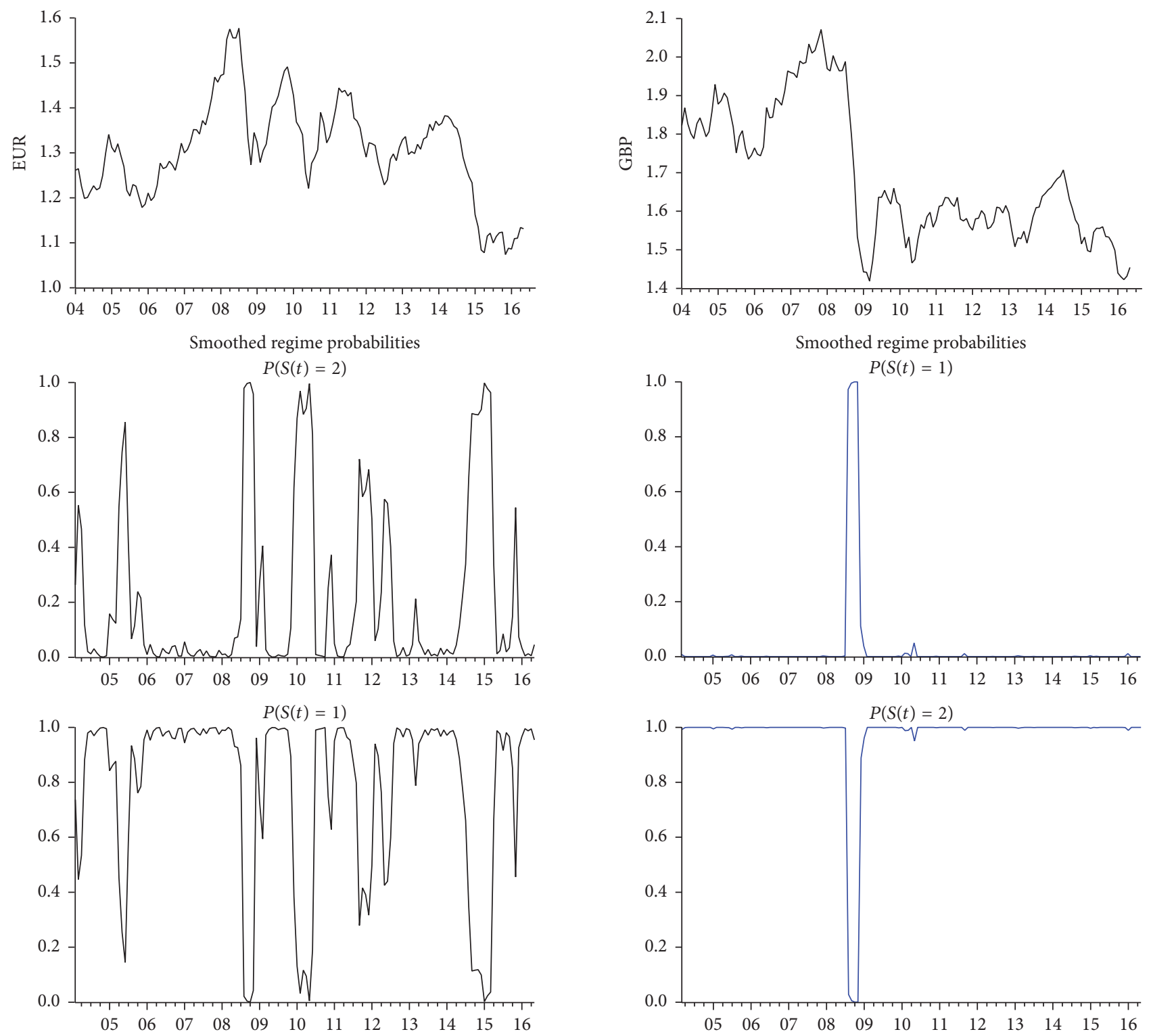

FIGURE 3: Time and smoothed probability plots of USD/EUR for 2004M01-2016M05.

trend in the middle panels of Figures 3 and 4 . In all, the Markov switching model gave patterns that were consistent with the corresponding series.

3.1.2. Case $k=3$. Three states could be identified: the depreciation (state 1), high appreciation (state 2), and low appreciation (state 3 ). The mean statistics $\widehat{\mu}$ showed that, in state 1 , Nigerian naira fell by $8.6 \%$ while states 2 and 3 correspond to monthly appreciation rates of .094\% and .0042\%, respectively. It is evident that state 1 experienced more volatility than the rest.

The point estimates of the transition probability are

$$
P=\left(\begin{array}{ccc}
0.400255 & 0.219180 & 0.380565 \\
1.19 \times 10^{-10} & 0.931781 & 0.068219 \\
0.043598 & 0.050775 & 0.905627
\end{array}\right)
$$

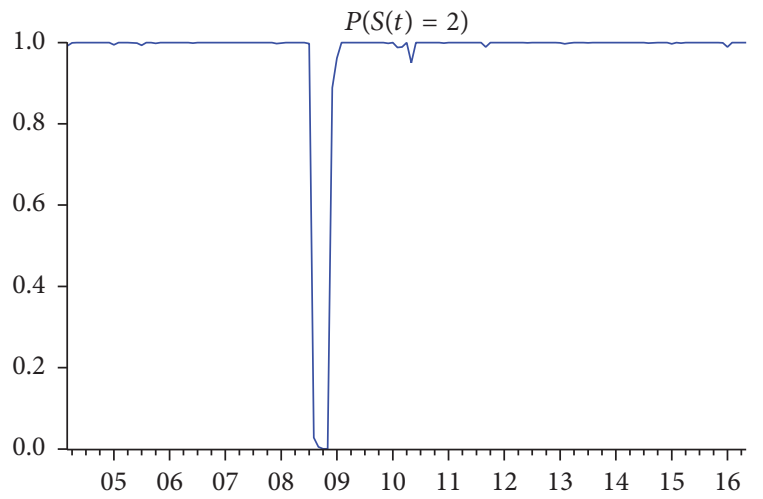

FIGURE 4: Time and smoothed probability plots of USD/GBP for 2004M01-2016M05.

The regime probabilities generated were $0.400,0.906$, and 0.932 for the depreciation, low appreciation and high appreciation states, respectively. This indicates that the exchange rate system has greater likelihood of remaining in states 2 and 3 than in state 1 . The expected duration estimates confirmed this as state 1 is expected to last for just one month while states 2 and 3 have duration of approximately fifteen and eleven months, respectively. A possible explanation for this is that the Nigerian monetary authorities would usually peg naira against dollar in the face of consistent depreciation thereby forcing the system into state 2 ; such was the case in the early 2015. $\widehat{P}$ also showed that, for a managed exchange rate system, the most unlikely transition is from the high appreciation state to depreciation, only a slight chance of $0.0000000119 \%$. For euros, pounds, and yen which are allowed to float, currencies may evolve freely from appreciation to depreciation 

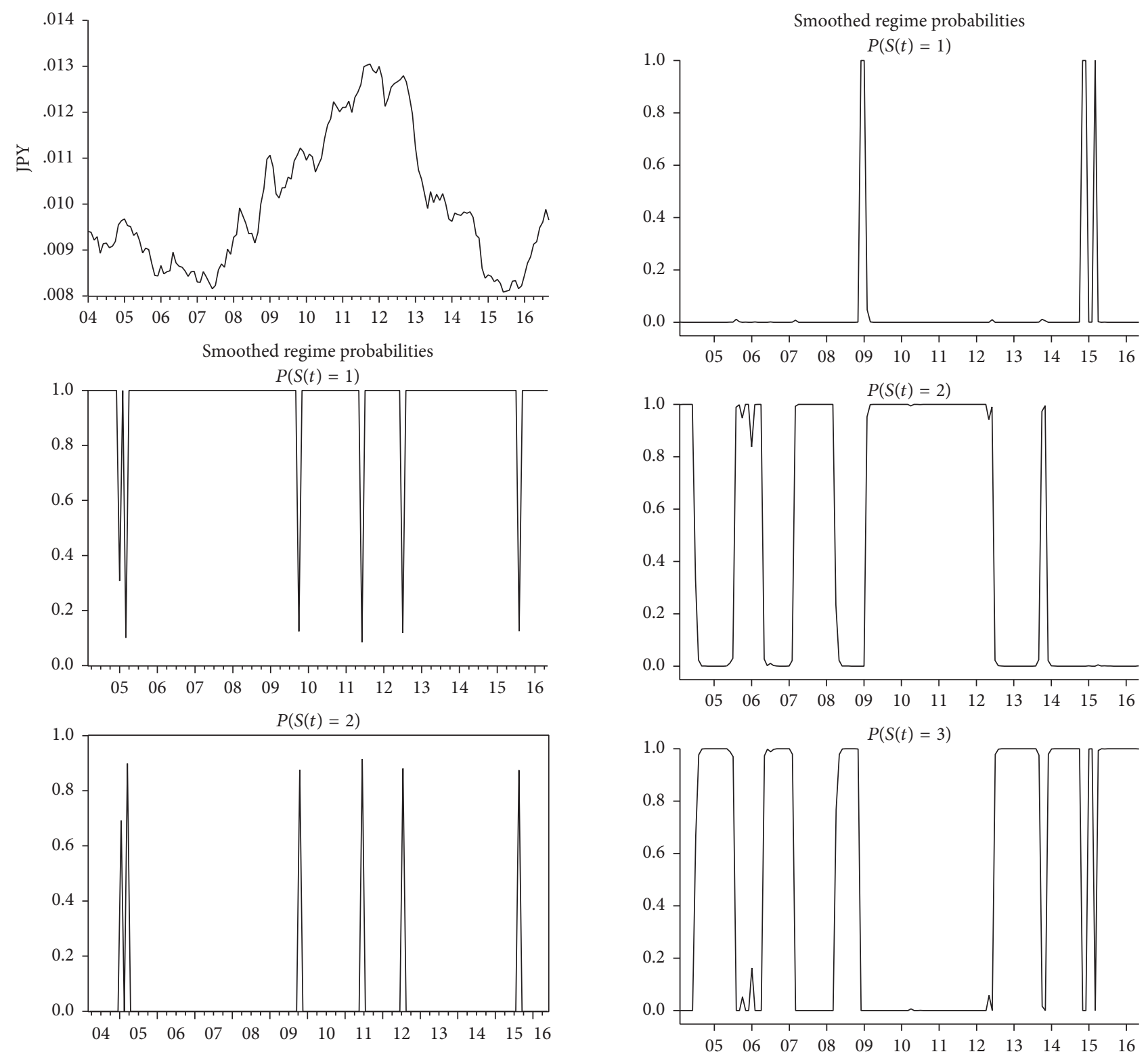

FIGURE 5: Time and smoothed probability plots of USD/JPY for 2004M01-2016M05.

and vice versa. On the other hand, $\widehat{P}_{12}=0.219$ implies that there is greater probability that the system enters into high appreciation state after depreciation. Such was the case between 2008 and 2009.

Now, along the line of Engel and Hamilton [2], we may define three possibilities for case $k=3$ :

(i) A random walk, that is, $P_{11}+P_{22}+P_{33}=1$, where exchange rate movements are independent from period to period

(ii) Long swings, that is, $\mu_{1}<0$ and $\left(\mu_{2}, \mu_{3}\right)>0$, and the values of $P_{j j} ; j=1,2,3$ are large

(iii) Asymmetric persistence in swings, that is, $\mu_{1}<0$ and $\left|\mu_{1}\right|$ is small and $P_{11}$ is large; in addition, $\left(\mu_{2}, \mu_{3}\right)>0$ and is large and $\left(P_{22}, P_{33}\right)$ is small

FIGURE 6: Smoothed probability plots of USD/NGN for 2004M012016M05.

Thus, from the estimates of $\mu$ and $\sigma$ coupled with those of the probabilities, it appears that the Nigerian exchange rates are characterized by asymmetric swings. That is, we have a case of $\widehat{\mu}_{1}$ negative and large in absolute, and $\widehat{P}_{11}$ small; together with $\left(\widehat{\mu}_{2}, \widehat{\mu}_{3}\right)$ positive and small, and $\left(\widehat{P}_{22}, \widehat{P}_{33}\right)$ large. The implication is that upward moves were gradual and drawn out while downward moves were short and sharp.

Lastly, the smoothed probabilities for the three states are displayed in Figure 6. We noticed that the smoothed probability plots divided Nigerian exchange rates into three segments that are consistent with the patterns in Figure 2. In particular, under state 1, Markov switching model identified two depreciation periods at 2008M12-2009M02 and 2014M112015M03. We recall that the first period was due to the US crisis that stemmed from the bankruptcy of the Lehman 
Brothers, which occurred around 2007-2008, and the effect spread to Nigerian currency market towards the tail end of 2008 and lasted for three months. The second period was due to the current fall in crude oil prices in late 2014. Since Nigerian economy heavily depends on the proceeds from the sale of crude and also the official exchange rate for crude oil is US dollars, it is no surprise, therefore, that the effects of the falling oil prices were felt almost immediately and significantly.

\subsubsection{Hypothesis Testing for a k-State Regime Model: Random} Walk versus Long Swings. We note, in passing, that some of the parameter estimates in Table 2 were not significant at $\alpha=0.05$. This should not generate a major concern since, in long swings test, interest is usually not in the individual $p$ values as they have no connotations other than the obvious fact that they are (in)significant. Rather attention and concern are in the test of random walk which combines the estimates and their standard errors in a meaningful fashion. This is the essence of long swings hypothesis. Thus, for the validity of our model, a natural test of hypothesis is to examine the claim that exchange rate follows a random walk. Engel and Hamilton [2] described well the procedure for a two-state regime model. However, since a three-state model for exchange rate cycle is first considered here (to our knowledge), it is therefore appropriate that we demonstrate how to adapt the test to 3 states or any number of state $k$ in general.

For a random walk, a general null hypothesis is

$$
\begin{aligned}
H_{0}: \sum_{j=1}^{k} P_{j j} & =1 \\
\mu_{1} & \neq \mu_{2} \neq \cdots \mu_{k} \\
\sigma_{1} & \neq \sigma_{2} \neq \cdots \sigma_{k},
\end{aligned}
$$

where $k$ is the number of states. System (15) implies that the state variables are independent. That is, under $H_{0}$, exchange rate movements are characterized by independent and identically distributed individual densities given by

$$
\begin{aligned}
P\left(r_{t} ; \theta\right)= & \frac{P_{11}}{\sqrt{2 \pi} \sigma_{1}} \exp \left(-\frac{1}{2}\left(\frac{r_{t}-\mu_{1}}{\sigma_{1}}\right)^{2}\right) \\
& +\frac{P_{22}}{\sqrt{2 \pi} \sigma_{2}} \exp \left(-\frac{1}{2}\left(\frac{r_{t}-\mu_{2}}{\sigma_{2}}\right)^{2}\right)+\cdots \\
& +\frac{\left(1-\sum_{j=1}^{(k-1)} P_{j j}\right)}{\sqrt{2 \pi} \sigma_{k}} \exp \left(-\frac{1}{2}\left(\frac{r_{t}-\mu_{k}}{\sigma_{k}}\right)^{2}\right) .
\end{aligned}
$$

Thus, one can test $H_{0_{p}}: P_{11}+P_{22}+\cdots+P_{k k}=1$ against the alternative hypothesis that $P_{11}+P_{22}+\cdots+P_{k k} \neq 1$, using standard theory of distribution since the parameter vector $\theta=\left(\mu_{1}, \mu_{2}, \ldots, \mu_{k}, \sigma_{1}, \sigma_{2}, \ldots, \sigma_{k}\right)$ and probabilities $P_{11}, P_{22}, \ldots, P_{k k}$ are identified under $H_{0_{P}}$.

For Wald test, therefore, we have the statistic

$$
W_{P}=\frac{\left(\sum_{j=1}^{k} \widehat{P}_{j j}-1\right)^{2}}{\sum_{i=1}^{k} \sum_{j=1}^{k} \operatorname{Cov}\left(\widehat{P}_{i i}, \widehat{P}_{j j}\right)} \sim \chi_{(1)}^{2} .
$$

TABLE 3: Hypotheses testing: random walk versus long swings.

\begin{tabular}{lcccc}
\hline \multirow{2}{*}{ Statistic } & \multicolumn{4}{c}{ Currencies } \\
& EUR & GBP & JPY & NGN \\
\hline \multirow{2}{*}{$W_{P}$} & 28.153 & 2.244 & $1.87 \times 10^{-6}$ & 177.973 \\
& $(0.000)$ & $(0.134)$ & $(0.999)$ & $(0.000)$ \\
\multirow{2}{*}{$W_{\mu}$} & 13.697 & 6.317 & 0.801 & 9.990 \\
& $(0.0002)$ & $(0.012)$ & $(0.371)$ & $(0.007)$ \\
\hline
\end{tabular}

$p$ values in parentheses.

The second test

$$
H_{0_{\mu}}: \mu_{1}=\mu_{2}=\cdots=\mu_{k}
$$

can be rewritten as

$$
H_{0_{\mu}}: R \mu=0,
$$

where $R$ is an $\left(\begin{array}{c}k \\ 2\end{array}\right) \times k$ matrix and $\mu$ is a $k \times 1$ vector. Then, the corresponding Wald statistic, $W_{\mu}$, is

$$
W_{\mu}=(R \widehat{\mu})^{\prime}\{R \operatorname{Cov}(\widehat{\mu})\}(R \widehat{\mu}) \sim \chi_{\left.\left({ }_{2}^{k}\right)\right)}^{2},
$$

where $\operatorname{Cov}(\mu)$ is the variance-covariance matrix of $\mu$.

For $k=2$, for instance, $W_{\mu}$ reduces to Engel and Hamilton's [2] equation for testing $H_{0}^{\prime \prime}$ (pg 699). For a particular case $k=3, R=\left(\begin{array}{ccc}1 & -1 & 0 \\ 0 & -1 & 1\end{array}\right), \mu=\left(\begin{array}{lll}\mu_{1} & \mu_{2} & \mu_{3}\end{array}\right)^{\prime}$, and $W_{\mu} \sim \chi_{(2)}^{2}$.

Table 3 presented Wald test statistics $W_{P}$ and $W_{\mu}$. At $\alpha=$ 0.05 , the critical values for $\chi_{(1)}^{2}$ and $\chi_{(2)}^{2}$ are 3.84 and 5.99, respectively. Since $28.2>3.84$ and $13.7>3.84$, we reject null hypothesis of random walk in favour of long swings for the euros and conclude that USD/EUR exhibited symmetric long swings between 2004M01 and 2016M05. This agrees with the visuals in Figure 1. Further, since $177.973>3.84$ and $9.990>$ 5.99 , we also reject the null in favour of asymmetric swings in the case of NGN. We therefore conclude that the naira experienced long, gradual swings in appreciation and short, sharp swings in depreciation. This is, of course, consistent with Figure 2 as the maximum duration for depreciation was 6 months, that is, 2014M10-2015M03, as opposed to that of appreciation which had a maximum duration of 15 months being from 2004M05 to 2005M07.

Test values on GBP presented mixed results while those of YEN were altogether not significant. Thus, we fail to reject $H_{0_{P}}$ and $H_{0_{\mu}}$ in the cases of Japanese yen and British pounds. Klaassen [3] however cautioned that one should not be too quick to conclude on the absence of long swings as statistical evidence depends heavily on data frequency and the period under consideration. He made this remark while experimenting with quarterly, monthly and weekly data frequencies of Japanese yen, British pounds and German mark from April 1974 to July 2003. He noted that, from the second quarter of 1974 (1974:II) through 1986:IV, statistical tests provided evidence in support of long swings hypothesis for the three currencies; however, when the data range was extended to 2003:II, all statistic values became insignificant at $\alpha=0.05$, 
TABLE 4: Model diagnostics.

\begin{tabular}{lcccccccc}
\hline \multirow{2}{*}{ Statistic } & \multicolumn{4}{c}{ Before MSM } & \multicolumn{3}{c}{ After MSM } & \multicolumn{2}{c}{ GBP } & JPY & NGN \\
& EUR & GBP & JPY & NGN & EUR & GBP & 9.057 & 3.888 \\
\multirow{2}{*}{$Q(10)$} & 26.428 & 10.311 & 8.584 & 12.906 & 13.085 & 9.696 & $(0.527)$ & $(0.952)$ \\
& $(0.003)$ & $(0.414)$ & $(0.572)$ & $(0.229)$ & $(0.219)$ & $(0.468)$ & $(0.527)$ \\
\multirow{2}{*}{$Q^{2}(10)$} & 20.868 & 21.586 & 8.403 & 2.163 & 25.277 & 19.640 & 6.124 & 0.276 \\
& $(0.022)$ & $(0.017)$ & $(0.590)$ & $(0.995)$ & $(0.005)$ & $(0.033)$ & $(0.805)$ & $(1.000)$ \\
\hline
\end{tabular}

MSM means Markov switching model. $p$ values are in parentheses.

casting doubts on earlier evidence of long swings. Along this line of thought, we note that the period between 2004 and 2016 gave too few observations to distinguish upward swings from a random walk in the cases of USD/GBP and USD/YEN (see top panels of Figures 4 and 5). Consequently, for that period, we conclude that exchange rates did not provide convincing evidence to reject the null hypothesis of random walk for Japanese yen and British pounds.

3.2. Model Diagnostics. This section concerns model (mis)specification. We tested for evidence of "left-over" serial correlation and $\mathrm{ARCH}$ effects in the residuals of the Markov switching models. Table 4 reported the Ljung-Box Q-statistic for standardized and squared standardized residuals at lag 1 through 10 (denoted $Q(10)$ and $\left.Q^{2}(10)\right)$ for the four models.

It is known in general that if a model is correctly specified, then the residuals should be free of serial correlation. We observed that $Q(10)$ values for all series were not significant at $\alpha=0.05$, which indicates the absence of serial correlation from estimated residuals. We observed further that $p$ values improved substantially after exchange rate returns were modeled with the Markov-modulated regime models.

Further, since the seminal work of Engle [10], it has become a custom to test financial time series for the presence of autoregressive conditional heteroscedasticity (ARCH) effects. So we implemented ARCH test using the serial correlation features of $\widehat{\epsilon}^{2}$. We found some evidence of ARCH effects for EUR and GBP but may not consider it significant at $\alpha=0.05$ given the number of observations. Otherwise, Markov switching models which incorporate GARCH specification in the variance equation have been developed. This may be employed to improve robustness of results.

\section{Conclusion}

The study developed a $k$-state $(k \geq 2)$ Markov switching model for the investigation of the long swings hypothesis in exchange rate movements. The model was applied to euros, British pounds, Japanese yen, and Nigerian naira. It is evident from the study that the choice of the number of states $k$ for exchange rates is currency-specific. As a matter of fact, the choice depends largely on the exchange rate regime adopted in each country, whether floating, fixed, or mixed. Currencies, such as euros, pounds and yen, which are exposed to a floating regime consistently would most likely be described by a two-state model, whereas one which switches between floating and fixed is a candidate for a three-state model. An example is the Nigerian naira.
Empirical results gave convincing evidence of long swings in euros and asymmetric swings in the USD/NGN rates; that is, Nigerian naira experienced long, gradual swings in appreciation and short, sharp swings in depreciation. Despite being insignificant, evidences of long swings and asymmetric swings were found in British pounds and Japanese yen, respectively. One way to explain this insignificance was discussed at length in Klaassen [3]. Samples of data may be too few to provide evidence of long swings.

Another perspective was given in Rabah [11]: hypothesis testing on random walk versus long swings is problematic due to the fact that asymptotic theory is difficult to establish under Markov regime modeling (see also Hansen $[12,13]$ ). Consequently, the study suggested bootstrap procedure to evaluate the statistical significance of Markov switching models. In a wider sense, bootstrapping techniques have been suggested as a means to solving the bias problem encountered in autoregressive (Kim [14]), mixed logit (Tsagkanos [15]), or, in general, maximum likelihood (Rabah [11]) estimators. Thus, further research can be carried out by exploring this avenue for a more robust estimation and inference in Markov switching modeling.

\section{Competing Interests}

The author declares that there is no conflict of interests regarding the publication of this paper.

\section{References}

[1] J. D. Hamilton, "A new approach to the economic analysis of nonstationary time series and the business cycle," Econometrica, vol. 57, no. 2, pp. 357-384, 1989.

[2] C. Engel and J. Hamilton, "Long swings in the dollar: are they in the data and do market know it?" American Economic Review, vol. 80, pp. 687-713, 1990.

[3] F. Klaassen, "Long swings in exchange rates: are they really in the data?" Journal of Business \& Economic Statistics, vol. 23, no. 1, pp. 87-95, 2005.

[4] R. Garcia and P. Perron, "An analysis of the real interest rate under regime shifts," Review of Economics and Statistics, vol. 78, no. 1, pp. 111-125, 1996.

[5] R. A. Meese and K. Rogoff, "Empirical exchange rate models of the seventies: do they fit out of sample?" Journal of International Economics, vol. 14, no. 1-2, pp. 3-24, 1983.

[6] G. Kaminsky, "Is there a peso problem? Evidence from the dollar/pound exchange rate, 1976-1987," American Economic Review, vol. 83, pp. 450-472, 1993. 
[7] J. D. Hamilton, Time Series Analysis, Princeton University Press, Princeton, NJ, USA, 1994.

[8] C.-J. Kim, "Dynamic linear models with Markov-switching," Journal of Econometrics, vol. 60, no. 1-2, pp. 1-22, 1994.

[9] J. Kim and C. Nelson, State-Space Models with Regime Switching, MIT Press, Cambridge, Mass, USA, 1999.

[10] R. F. Engle, "Autoregressive conditional heteroscedasticity with estimates of the variance of United Kingdom inflation," Econometrica, vol. 50, no. 4, pp. 987-1007, 1982.

[11] Z. Rabah, "Does bootstrap testing perform well for Markov switching models?" in Proceedings of the 6th Eurostat Colloquium on Modern Tools for Business Cycle Analysis: The Lessons from Global Economic Crisis, vol. EWP2011/025, Luxembourg City, Luxembourg, 2010.

[12] B. E. Hansen, "The likelihood ratio test under nonstandard conditions: testing the markov switching model of gnp," Journal of Applied Econometrics, vol. 7, pp. S61-S82, 1992.

[13] B. E. Hansen, "Erratum: the likelihood ratio test under nonstandard conditions: testing the Markov switching model of GNP," Journal of Applied Econometrics, vol. 11, no. 2, pp. 195-198, 1996.

[14] J. H. Kim, "Bias-correction and endogenous lag order algorithm for bootstrap prediction intervals," Journal of Statistical Planning and Inference, vol. 177, pp. 41-44, 2016.

[15] A. G. Tsagkanos, "A bootstrap-based minimum bias maximum simulated likelihood estimator of Mixed Logit," Economics Letters, vol. 96, no. 2, pp. 282-286, 2007. 


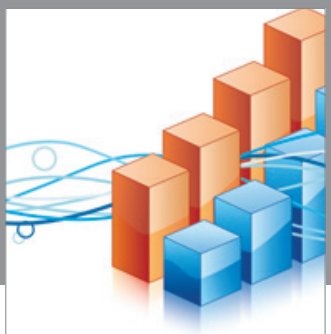

Advances in

Operations Research

vatem alat4



\section{The Scientific} World Journal

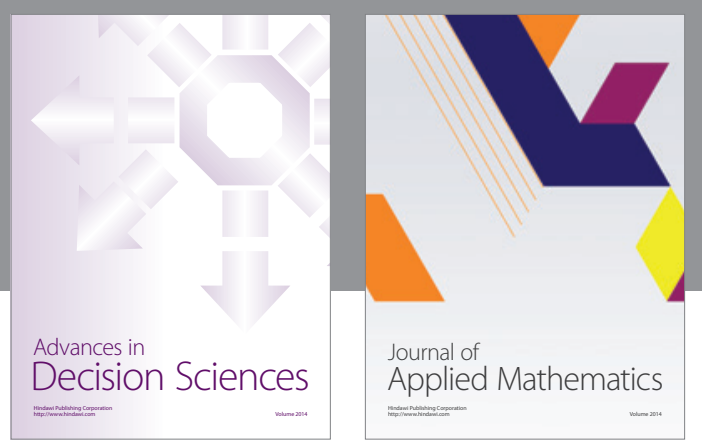

Algebra

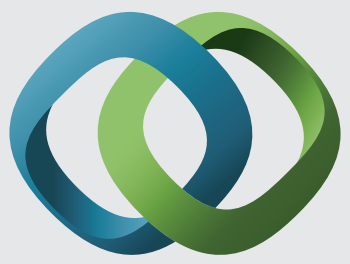

\section{Hindawi}

Submit your manuscripts at

http://www.hindawi.com
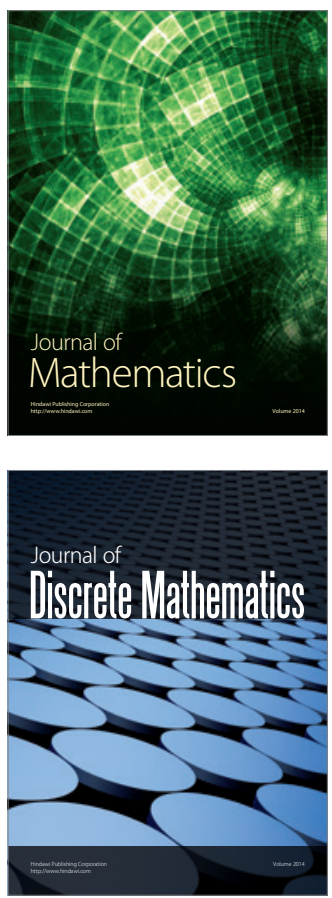

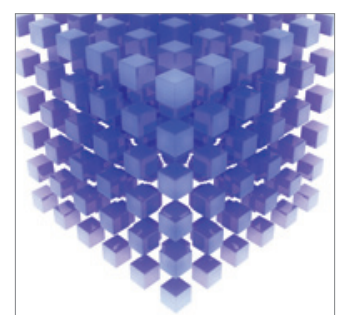

Mathematical Problems in Engineering
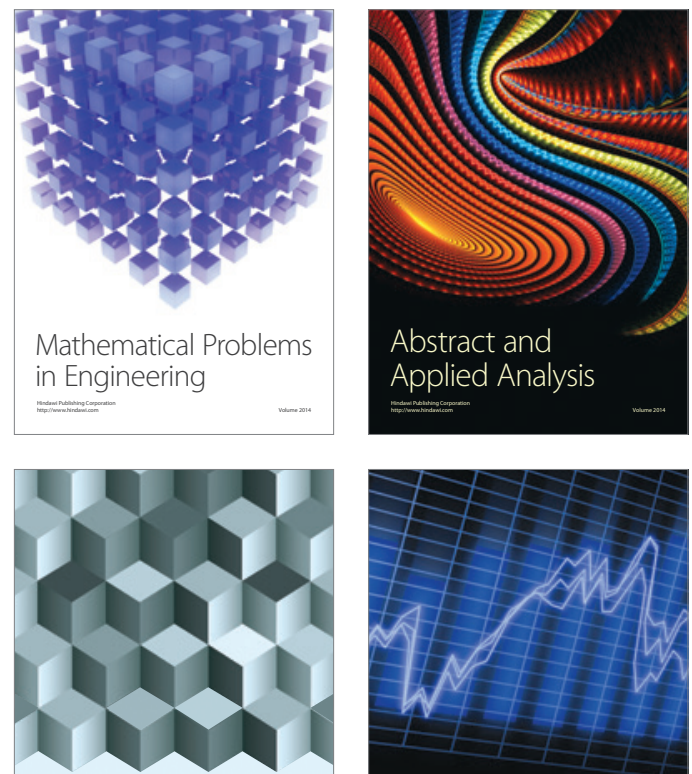

Journal of

Function Spaces

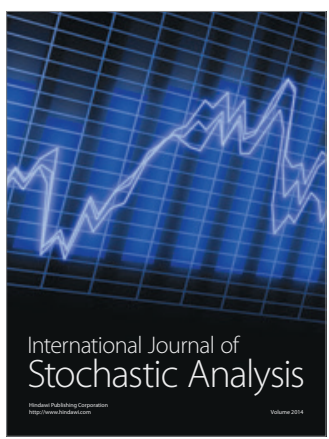

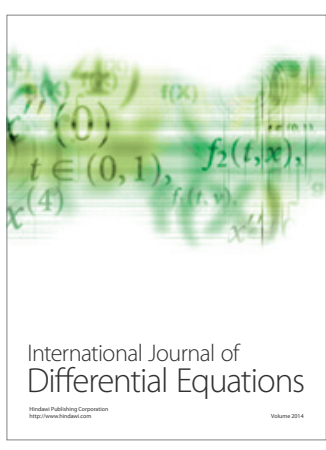
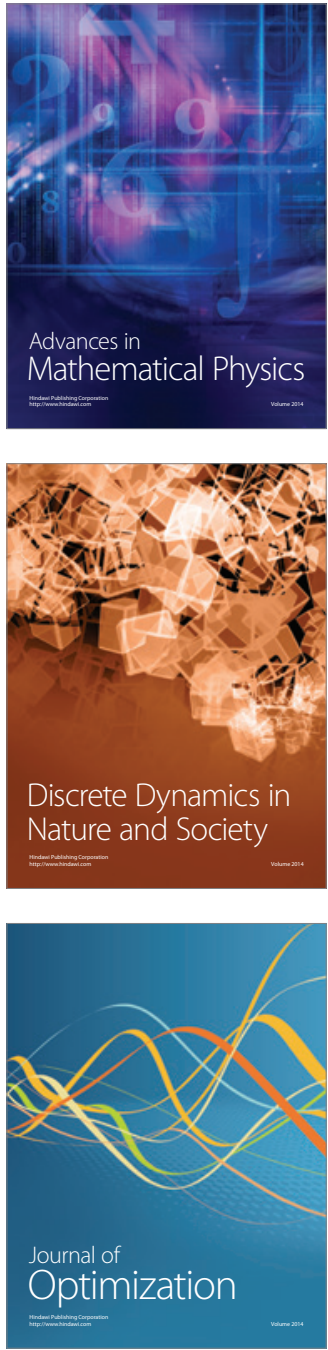